\title{
A Boundary Integral Equation Related to the Ahlfors Map
}

\author{
Nur H. A. A. Wahid ${ }^{\text {, }}$ Ali H. M. Murid ${ }^{\mathrm{b}, *}$, Mukhiddin I. Muminov ${ }^{\mathrm{c}}$ \\ ${ }^{a}$ Faculty of Computer and Mathematical Sciences, Universiti Teknologi MARA, 40450 \\ Shah Alam, Selangor, Malaysia; 'bepartment of Mathematical Sciences, Faculty of \\ Science, Universiti Teknologi Malaysia, 81310 Johor Bahru, Malaysia; 'Mathematical \\ Faculty, Samarkand State University, 140104, Samarkand, Uzbekistan
}

Abstract The Ahlfors map is a mapping function that maps a multiply connected region onto a unit disk. This paper presents a new boundary integral equation related to the Ahlfors map of a bounded multiply connected region. The boundary integral equation is constructed from a boundary relationship satisfied by the Ahlfors map of a multiply connected region.

Keywords: Ahlfors map, multiply connected region, Kerzman-Stein kernel, Neumann kernel

\section{Introduction}

The Ahlfors map is a generalization of the Riemann map for a simply connected region. For a multiply connected region $\Omega$ of connectivity $M+1, M=1,2, \ldots$, the Ahlfors map with the base point $a \in \Omega$ is a $M+1$-to-one map. It maps each boundary component of $\Omega$ one-to-one onto the unit disk and maps $a$ to the origin [1, 2, 3]. The Ahlfors map might prove to be useful in the problem of fluid mechanics [1]. Bell [1] computed the Ahlfors map for a bounded multiply connected region without the knowledge of the zeros of the Ahlfors map. The method in [1] is based on the generalization of the Kerzman-Stein method in [4]. In addition, [2, 3] also computed the Ahlfors map for an annulus region. Some integral equations related to the Ahlfors map have been constructed in [5-8]. The integral equations in [5, 7, 8] are based on the boundary relationship satisfied by an analytic function involving the Kerzman-Stein kernel, the Neumann kernel, the Neumann-type kernel and the Kerzman-Stein type kernel. The methods in [5-8] however depend on the zeros of the Ahlfors map.

In this paper, we present a new boundary integral equation method related to the Ahlfors map of a bounded multiply connected region different from [5-8] using a boundary relationship satisfied by the

${ }^{*}$ For correspondence: alihassan@utm.my

Received: 16 March 2020 Accepted: 30 June 2020

(C) Copyright Wahid. This article is distributed under the terms of the Creative Commons Attribution License, which permits unrestricted use and redistribution provided that the original author and source are credited.
Ahlfors map. The new boundary integral equation will be useful in computing the zeros of the Ahlfors map.

The organization of this paper is as follows: Section 2 presents some auxiliary materials related to the Ahlfors map. In Section 3 we give a new boundary integral equation related to the Ahlfors map for a bounded multiply connected region. In Section 4 we give a conclusion.

\section{Auxiliary materials}

Let $\Omega$ be a bounded multiply connected region with connectivity $M+1, M=1,2, \ldots$ consisting of $M+1$ smooth closed Jordan curves $\Gamma_{j}, j=0,1, \ldots, M$, i.e. $\Gamma=\Gamma_{0} \cup \Gamma_{1} \cup \cdots \Gamma_{M}$. The curves $\Gamma_{1}, \ldots, \Gamma_{M}$ lie in the interior of the outer boundary $\Gamma_{0}$. The inner curves $\Gamma_{1}, \ldots, \Gamma_{M}$ have clockwise orientations and the outer curve $\Gamma_{0}$ has a counterclockwise orientation as shown in Figure 2.1. 


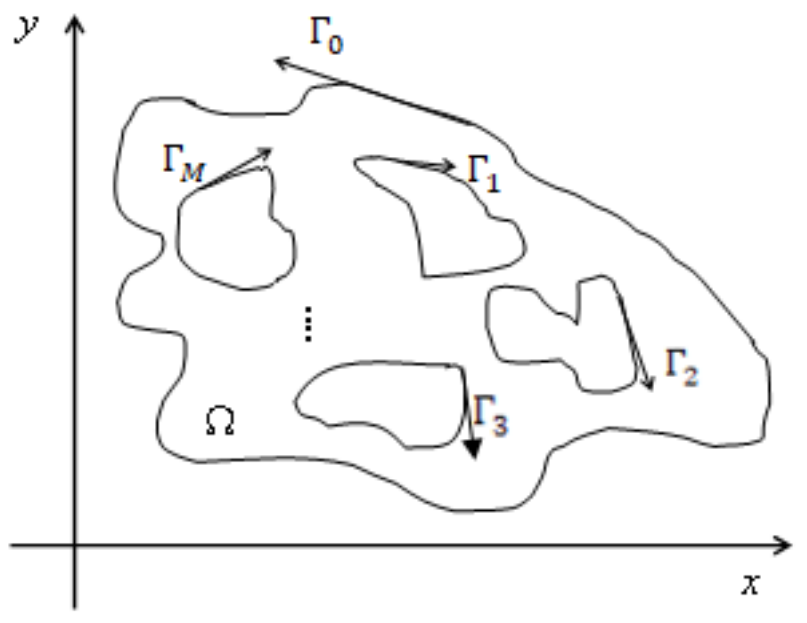

$z$-plane

Figure 2.1 A bounded multiply connected region $\Omega$

The curves $\Gamma_{j}, j=0,1, \ldots, M$ are parameterized by $2 \pi$-periodic twice continuously differentiable complex-valued functions $z_{j}(t)$ with non-vanishing first derivatives $z_{j}^{\prime}(t) \neq 0, t \in J_{j}=[0,2 \pi]$. The total parameter domain $J$ is defined as the disjoint union of $M+1$ intervals of $J_{j}, j=0,1, \ldots, M$. The parameterization $z(t)$ of the whole boundary $\Gamma$ on $J$ is defined as $z(t)=z_{j}(t), t \in J_{j}=[0,2 \pi]$. The unit tangent to the boundary $\Gamma_{j}$ at $z_{j}(t)$ is given by

$T\left(z_{j}(t)\right)=\frac{z_{j}^{\prime}(t)}{\left|z_{j}^{\prime}(t)\right|}$.

The Szegö kernel is known to satisfy the Kerzman-Stein integral equation [4, 9]

$S\left(z, a_{0}\right)+\int_{\Gamma} A(z, w) S\left(w, a_{0}\right)|d w|=g(z), \quad a_{0} \in \Omega$,

where

$A(z, w)= \begin{cases}\frac{1}{2 \pi i}\left(\frac{T(w)}{z-w}-\frac{\overline{T(z)}}{\bar{z}-\bar{w}}\right), & z \neq w \in \Gamma, \\ 0, & z=w \in \Gamma,\end{cases}$

$g(z)=-\frac{1}{2 \pi i} \frac{\overline{T(z)}}{\bar{z}-\overline{a_{0}}}$.

The function $A(z, w)$ is known as the Kerzman-Stein kernel and it is continuous on the smooth boundary of $\Omega[4,10]$. It has the property that $A(z, w)=0$ if $z, w$ are on a circle. Numerical implementation of computing the Szegö kernel based on (2.2) is discussed in [10]. With $z=z(t)$ and $w=z(s),(2.2)$ becomes

$S\left(z(t), a_{0}\right)+\int_{J} A(z(t), z(s)) S\left(z(s), a_{0}\right)\left|z^{\prime}(s)\right| d s=g(z(t))$.

The derivative of the Szegö kernel has been derived in [7] as a solution of the integral equation 


$$
\begin{aligned}
& S^{\prime}\left(z(t), a_{0}\right) z^{\prime}(t)=g^{\prime}(z(t)) z^{\prime}(t) \\
& -\int_{J}\left[\frac{d}{d t} A(z(t), z(s)) z^{\prime}(t)\right] S\left(z(s), a_{0}\right)\left|z^{\prime}(s)\right| d s,
\end{aligned}
$$

where

$$
\begin{aligned}
& g^{\prime}(z(t)) z^{\prime}(t)=-\frac{1}{2 \pi i}\left[\frac{\overline{T^{\prime}(z(t)) z^{\prime}(t)}}{\overline{z(t)-a_{0}}}-\frac{\overline{T(z(t)) z^{\prime}(t)}}{\overline{\left(z(t)-a_{0}\right)^{2}}}\right], \\
& T^{\prime}(z(t)) z^{\prime}(t)=\frac{z^{\prime \prime}(t)}{2\left|z^{\prime}(t)\right|}-\frac{\left(z^{\prime}(t)\right)^{2} \overline{z^{\prime \prime}(t)}}{2\left|z^{\prime}(t)\right|^{3}},
\end{aligned}
$$

and

$$
\begin{aligned}
& \int \frac{1}{2 \pi i}\left[\frac{-T(z(s)) z^{\prime}(t)}{(z(t)-z(s))^{2}}-\frac{\overline{T^{\prime}(z(t)) z^{\prime}(t)}}{\overline{(\overline{z(t)}-\overline{z(s)})}}\right.
\end{aligned}
$$

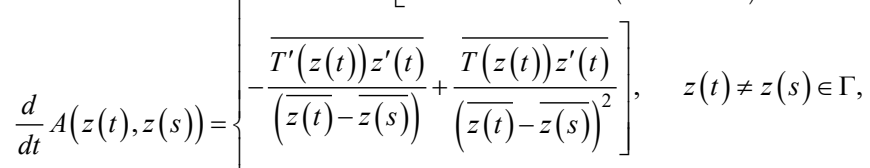

$$
\begin{aligned}
& \frac{1}{4 \pi \mid z^{\prime}(t)}\left[\frac{1}{3} \operatorname{Im}\left(\frac{z^{\prime \prime \prime}(t)}{z^{\prime}(t)}\right)\right. \\
& \left.-\operatorname{Re}\left(\frac{z^{\prime \prime}(t)}{z^{\prime}(t)}\right) \operatorname{Im}\left(\frac{z^{\prime \prime}(t)}{z^{\prime}(t)}\right)\right], \quad z(t)=z(s) \in \Gamma .
\end{aligned}
$$

Let $f(z)$ be the Ahlfors function which maps $\Omega$ conformally onto the unit disk $E=\{w:|w|<1\}$ which satisfies the conditions $f\left(a_{j}\right)=0, j=0,1, \ldots, M$ and $f^{\prime}\left(a_{0}\right)>0$, where $a_{j} \in \Omega$ are the zeros of the Ahlfors map and $a_{0} \in \Omega$ can be freely chosen. The boundary values for $f(z)$ is represented by $f\left(z_{j}(t)\right)=e^{i \theta_{j}(t)}, \quad \Gamma_{j}: z=z_{j}(t), \quad 0 \leq t \leq 2 \pi \in J_{j}$,

where $\theta_{j}(t), j=0,1, \ldots, M$ are the boundary correspondence functions of the Ahlfors map on $\Gamma_{j}$. Differentiating both sides of (2.5), we have

$f^{\prime}\left(z_{j}(t)\right) z_{j}^{\prime}(t)=f\left(z_{j}(t)\right) i \theta_{j}^{\prime}(t)$.

Taking modulus on both sides of (2.6) gives

$\left|f^{\prime}\left(z_{j}(t)\right) z_{j}^{\prime}(t)\right|=\left|f\left(z_{j}(t)\right) i \theta_{j}^{\prime}(t)\right|$.

(2.7)

Dividing (2.6) by (2.7) and using (2.1), we get

$f\left(z_{j}(t)\right)=\frac{T\left(z_{j}(t)\right)}{i} \frac{\left|\theta_{j}^{\prime}(t)\right| f^{\prime}\left(z_{j}(t)\right)}{\theta_{j}^{\prime}(t)\left|f^{\prime}\left(z_{j}(t)\right)\right|}$.

The image of $\Gamma_{0}$ remains in a counterclockwise orientation, so $\theta_{0}^{\prime}(t)>0$ and the images of $\Gamma_{j}$, $j=1,2, \ldots, M$ are in reversed orientations, so $\theta_{j}^{\prime}(t)>0$.

Since $\frac{\left|\theta_{j}^{\prime}(t)\right|}{\theta_{j}^{\prime}(t)}=1,(2.8)$ becomes

$f\left(z_{j}(t)\right)=\frac{T\left(z_{j}(t)\right) f^{\prime}\left(z_{j}(t)\right)}{i\left|f^{\prime}\left(z_{j}(t)\right)\right|}$.

In general, 
$f(z)=\frac{T(z) f^{\prime}(z)}{i\left|f^{\prime}(z)\right|}, \quad z \in \Gamma$.

The interior values of the Ahlfors map $f(z)$ which is analytic on $\Omega$ can be obtained by using the Cauchy integral formula

$f(z)=\frac{1}{2 \pi i} \int_{\Gamma} \frac{f(w)}{w-z} d w, \quad z \in \Omega$.

For points $z$ closed to $\Gamma$, the method of Helsing and Ojala [11] can be used for the efficient numerical computation of (2.10).

It is known that the Ahlfors map can be represented in terms of the Szegö kernel $S\left(z, a_{0}\right)$ and the Garabedian kernel $L\left(z, a_{0}\right)$. It is given by [4]

$f(z)=\frac{S\left(z, a_{0}\right)}{L\left(z, a_{0}\right)}, \quad z \in \Omega \cup \Gamma, \quad a_{0} \in \Omega$.

Since $L\left(z, a_{0}\right)=i \overline{S\left(z, a_{0}\right) T(z)}$ [1], thus (2.11) can be written as

$f(z(t))=\frac{S\left(z(t), a_{0}\right) T(z(t))}{i \overline{S\left(z(t), a_{0}\right)}}, \quad z(t) \in \Gamma$.

Thus the boundary values for the Ahlfors map can be determined completely from the boundary values of the Szegö kernel.

Differentiating both sides of (2.12), we get

$$
\begin{aligned}
f^{\prime}(z(t)) z^{\prime}(t)= & \frac{1}{i}\left[\frac{S\left(z(t), a_{0}\right) T^{\prime}(z(t)) z^{\prime}(t)}{\overline{S\left(z(t), a_{0}\right)}}\right. \\
& +\frac{S^{\prime}\left(z(t), a_{0}\right) z^{\prime}(t) T(z(t))}{\overline{S\left(z(t), a_{0}\right)}} \\
& \left.-\frac{\overline{S^{\prime}\left(z(t), a_{0}\right) z^{\prime}(t) S\left(z(t), a_{0}\right) T(z(t))}}{\left(\overline{S\left(z(t), a_{0}\right)}\right)^{2}}\right] .
\end{aligned}
$$

In $[12,13]$ an alternative formula for the derivative of the boundary correspondence function of the Ahlfors map has been derived by using (2.6), (2.12) and (2.13), i.e.

$$
\theta^{\prime}(t)=2 \operatorname{Im}\left[\frac{S^{\prime}\left(z(t), a_{0}\right) z^{\prime}(t)}{S\left(z(t), a_{0}\right)}\right]+\operatorname{Im}\left[\frac{z^{\prime \prime}(t)}{z^{\prime}(t)}\right] \text {. }
$$

Suppose that $b(z), Q(z)$ and $H(z)$ are complex-valued functions defined on $\Gamma$ such that $b(z) \neq 0$, $Q(z) \neq 0, H(z) \neq 0$ and $\frac{\overline{H(z)}}{T(z) Q(z)}$ satisfies the Hölder condition on $\Gamma$, where $T(z)$ is the unit tangent vector on $\Gamma$. The function $P(z)$ is said to satisfy the interior relationship if $P(z)$ is analytic in $\Omega, P(z) \neq 0$ on $\Gamma$, and Hölder continuous on $\Omega \cup \Gamma$, and satisfies the non-homogeneous boundary relationship [14, p. 33]

$$
P(z)=\frac{b(z) \overline{T(z) Q(z) P(z)}}{\overline{G(z)}}+\overline{H(z)}, \quad z \in \Gamma,
$$

where $G(z)$ is analytic in $\Omega$, Hölder continuous on $\Gamma$, and $G(z) \neq 0$ on $\Gamma$. The boundary relationship 
(2.15) also has the following equivalent form [14, p. 33]

$$
G(z)=\frac{\overline{b(z)} T(z) Q(z) P(z)^{2}}{|P(z)|^{2}}+\frac{G(z) H(z)}{\overline{P(z)}} .
$$

The following theorem gives an integral equation for an analytic function $P(z)$ satisfying the interior non-homogeneous boundary relationship (2.15) or (2.16).

Theorem 2.1. [14, p. 34] Let $u(z)$ and $v(z)$ be any complex-valued functions that are defined on $\Gamma$. If the function $P(z)$ satisfies the boundary relationship (2.15) or (2.16), then

$$
\begin{aligned}
& \frac{1}{2}\left[v(z)+\frac{u(z)}{T(z) Q(z)}\right] P(z)+\operatorname{PV} \int_{\Gamma} K(z, w) P(w)|d w| \\
& +b(z) u(z)\left[\sum_{a_{j} \in \Gamma} \operatorname{Res}_{w=a_{j}} \frac{P(w)}{(w-z) G(w)}\right]^{-}=-u(z) \overline{L_{\bar{R}}(z)}, \quad z \in \Gamma,
\end{aligned}
$$

where

$$
K(z, w)=\frac{1}{2 \pi i}\left[\frac{b(z) u(z)}{b(w)(\bar{w}-\bar{z}) \overline{Q(w)}}-\frac{v(z) T(w)}{w-z}\right],
$$

and

$$
\begin{aligned}
L_{\bar{R}}(z) & =-\frac{1}{2} \frac{H(z)}{Q(z) T(z)} \\
& +\mathrm{PV} \frac{1}{2 \pi i} \int_{\Gamma} \overline{\overline{b(w)}(w-z) Q(w) T(w)} d w .
\end{aligned}
$$

The symbol "-" in the superscript denotes the complex conjugate and the sum in (2.17) is over all those zeros $a_{0}, a_{1}, \ldots, a_{M}$ of $G(z)$ that lie inside $\Omega$. There is no residue term if the function $G(z)$ has no zeros in $\Omega$. Theorem 2.1 generalizes the result in [5] which is limited to a doubly connected region.

We next derive a new boundary integral equation related to the Ahlfors map of a bounded multiply connected region using Theorem 2.1.

\section{Integral equation related to the Ahlfors map}

The following theorem gives another integral equation satisfied by $\theta^{\prime}(t)$ where $\theta(t)$ is the boundary correspondence function of the Ahlfors map of a bounded multiply connected region onto a unit disk.

Theorem 3.1. For all $z \in \Gamma$, the function $\theta^{\prime}(t)$ of the Ahlfors map for the multiply connected region satisfies

$i \theta^{\prime}(s)+\frac{1}{\pi} \mathrm{PV} \int_{J} \frac{z^{\prime}(s)}{z(s)-z(t)} \theta^{\prime}(t) d t$

$=2 z^{\prime}(s) \sum_{j=0}^{M} \frac{1}{z(s)-a_{j}}$,

where $a_{j}, j=0,1, \ldots, M$ are the zeros of the Ahlfors map.

Proof. The Ahlfors map for a multiply connected region can be written as

$f(z)=\prod_{j=0}^{M}\left(z-a_{j}\right) g(z), \quad z \in \Omega$, 
where $g(z)$ is analytic in $\Omega$ and $g(z) \neq 0$ for all $z \in \Omega$. Applying log on both sides of (3.2) gives $\log f(z)=\sum_{j=0}^{M} \log \left(z-a_{j}\right)+\log g(z), \quad z \in \Gamma$.

Differentiate on both sides of (3.3), we get

$\frac{f^{\prime}(z)}{f(z)}=\sum_{j=0}^{M} \frac{1}{z-a_{j}}+\frac{g^{\prime}(z)}{g(z)}$

so that

$D(z)=\frac{f^{\prime}(z)}{f(z)}-\sum_{j=0}^{M} \frac{1}{z-a_{j}}=\frac{g^{\prime}(z)}{g(z)}$

is analytic in $\Omega$ and hence (3.5) can be written as

$\frac{f^{\prime}(z)}{f(z)}=D(z)+\sum_{j=0}^{M} \frac{1}{z-a_{j}}$,

Squaring both sides of (2.9) gives

$f(z)^{2}=-\frac{T(z)^{2} f^{\prime}(z)^{2}}{\left|f^{\prime}(z)\right|^{2}}$.

Since $\left|f^{\prime}(z)\right|^{2}=f^{\prime}(z) \overline{f^{\prime}(z)}$ and $f(z)=\frac{1}{\overline{f(z)}},(3.7)$ becomes

$\frac{\overline{f^{\prime}(z)}}{\overline{f(z)}}=-\frac{T(z)^{2} f^{\prime}(z)}{f(z)}$.

Taking conjugate to both sides of (3.8), we get

$\frac{f^{\prime}(z)}{f(z)}=-\overline{T(z)}^{2}\left(\overline{\frac{f^{\prime}(z)}{f(z)}}\right)$.

Substituting (3.6) into (3.9), we obtain

$$
\begin{aligned}
D(z)+\sum_{j=0}^{M} \frac{1}{z-a_{j}} & =-\overline{T(z)}^{2}\left[\overline{D(z)}+\sum_{j=0}^{M} \frac{1}{\bar{z}-\overline{a_{j}}}\right], \\
D(z) & =-\overline{T(z)}^{2} \overline{D(z)}-\sum_{j=0}^{M} \frac{\overline{T(z)}^{2}}{\bar{z}-\overline{a_{j}}}-\sum_{j=0}^{M} \frac{1}{z-a_{j}} .
\end{aligned}
$$

Letting $u(z)=0$ and $v(z)=2$ in Theorem 2.1 we have

$$
P(z)+\operatorname{PV} \int_{\Gamma} K(z, w) P(w)|d w|=0,
$$

where

$K(z, w)=-\frac{1}{2 \pi i}\left[\frac{2 T(w)}{w-z}\right]$.

Comparing (2.15) and (3.10) gives the

following

assignments

$P(z)=D(z), \quad Q(z)=1, \quad G(z)=1, \quad b(z)=-\overline{T(z)}$,

and

$H(z)=-\sum_{j=0}^{M} \frac{T(z)^{2}}{z-a_{j}}-\sum_{j=0}^{M} \frac{1}{\bar{z}-\bar{a}_{j}}$.

Substituting the above assignments into (3.11) and using (3.5), we obtain 
$\frac{f^{\prime}(z)}{f(z)}-\sum_{j=0}^{M} \frac{1}{z-a_{j}}-2 \mathrm{PV} \int_{\Gamma} \frac{1}{2 \pi i}\left[\frac{T(w)}{w-z}\right]\left[\frac{f^{\prime}(w)}{f(w)}-\sum_{j=0}^{M} \frac{1}{w-a_{j}}\right]|d w|=0$.

Rearranging (3.12) and since $T(w)|d w|=d w$, gives

$$
\begin{aligned}
& \frac{f^{\prime}(z)}{f(z)}-2 \mathrm{PV} \int_{\Gamma} \frac{1}{2 \pi i} \frac{1}{w-z} \frac{f^{\prime}(w)}{f(w)} d w \\
& +2 \mathrm{PV} \sum_{j=0}^{M} \int_{\Gamma} \frac{1}{2 \pi i} \frac{1}{w-z} \frac{1}{w-a_{j}} d w=\sum_{j=0}^{M} \frac{1}{z-a_{j}} .
\end{aligned}
$$

Using the fact that $[15$, p. 91]

$\mathrm{PV} \frac{1}{2 \pi i} \int_{\Gamma} \frac{1}{w-z} \frac{1}{w-a_{j}} d w=-\frac{1}{2\left(z-a_{j}\right)}$,

and after some simplifications (3.13) becomes

$\frac{1}{2} \frac{f^{\prime}(z)}{f(z)}-\mathrm{PV} \int_{\Gamma} \frac{1}{2 \pi i} \frac{1}{w-z} \frac{f^{\prime}(w)}{f(w)} d w=\sum_{j=0}^{M} \frac{1}{z-a_{j}}$.

Letting $z=z(s)$ and $w=z(t)$, and multiplying (3.14) by $z^{\prime}(s)$, gives

$$
\begin{aligned}
& \frac{1}{2} \frac{f^{\prime}(z(s)) z^{\prime}(s)}{f(z(s))} \\
& +\frac{1}{2 \pi i} \mathrm{PV} \int_{J} \frac{z^{\prime}(s)}{z(s)-z(t)} \frac{f^{\prime}(z(t))}{f(z(t))} z^{\prime}(t) d t=z^{\prime}(s) \sum_{j=0}^{M} \frac{1}{z(s)-a_{j}} .
\end{aligned}
$$

Applying (2.6) to (3.15) yields the integral equation (3.1).

This completes the proof of Theorem 3.1.

Observe that, by taking the imaginary part on both sides of (3.1), it reduces to the integral equation derived in [7], i.e.

$$
\theta^{\prime}(s)+\int_{J} N^{*}(s, t) \theta^{\prime}(t) d t=2 \operatorname{Im}\left[\sum_{j=0}^{M} \frac{z^{\prime}(s)}{z(s)-a_{j}}\right],
$$

where $[16,17]$

$$
N^{*}(s, t)= \begin{cases}\frac{1}{\pi} \operatorname{Im}\left[\frac{z^{\prime}(s)}{z(s)-z(t)}\right], & t \neq s, \\ \frac{1}{2 \pi} \operatorname{Im}\left[\frac{z^{\prime \prime}(s)}{z^{\prime}(s)}\right], & t=s,\end{cases}
$$

and $\theta^{\prime}(t)$ is defined in (2.14). The kernel $N^{*}(s, t)$ is the adjoint of the classical Neumann kernel $N(s, t)=N^{*}(t, s)$. It is well known that $\lambda=-1$ is an eigenvalue of the Neumann kernel $N(s, t)$ with multiplicity $M$ [17]. The eigenfunctions of $N(s, t)$ corresponding to the eigenvalue $\lambda=-1$ are $\left\{\chi^{[1]}, \chi^{[2]}, \ldots, \chi^{[M]}\right\}$, where [18] $\chi^{[k]}(\xi)= \begin{cases}1, & \xi \in \Gamma_{k}, k=1,2, \ldots, M, \\ 0, & \text { otherwise. }\end{cases}$

By the Fredholm alternative theorem [15], the integral equation (3.16) is solvable if and only if the given function $g(s)=2 \operatorname{Im}\left[\sum_{j=0}^{M} \frac{z^{\prime}(s)}{z(s)-a_{j}}\right]$ satisfies the $M$ orthogonality conditions 
$\left(g, \chi^{[k]}\right)=\int_{J} g(t) \chi^{[k]}(t) d t=0, \quad k=1,2, \ldots, M$.

Perhaps these $M$ orthogonality conditions could be used to find the $M$ unknown zeros $a_{1}, \ldots, a_{M}$ in the right-hand side of the integral equation (3.16). If $\Omega$ is a simply connected region, then (3.16) reduces to the Warschawski's integral equation for Riemann map as given in [15, p. 394-395] with $R(0)=0$, i.e.

$$
\theta^{\prime}(s)+\int_{J} N^{*}(s, t) \theta^{\prime}(t) d t=2 \operatorname{Im}\left[\frac{z^{\prime}(s)}{z(s)}\right]
$$

If is a doubly connected region, equation (3.1) reduces to the result given in [19] by a different approach.

\section{Conclusion}

In this paper, we have presented a new boundary integral equation (3.1) related to the Ahlfors map of a bounded multiply connected region which contains the special cases (3.16) and (3.17).

\section{Acknowledgements}

The first author would like to acknowledge the Ministry of Education Malaysia (MOE) for MyBrainSc scholarship. The authors also wish to thank the anonymous referees for their helpful suggestions.

\section{References}

[1] Bell S. R., 1986, Numerical computation of the Ahlfors map of a multiply connected planar domain, Journal of Mathematical Analysis and Applications, 120, 211-217.

[2] Tegtmeyer T. J., 1998, The Ahlfors Map and Szegö Kernel in Multiply Connected Domains, [Ph.D. thesis], Purdue University.

[3] Tegtmeyer T. J. and Thomas A. D., 1999, The Ahlfors map and Szegö kernel for an annulus, Rocky Mountain Journal of Mathematics, 29(2), 709-723.

[4] Kerzman N. and Stein E. M., 1978, The Cauchy kernel, the Szegö kernel, and the Riemann mapping function, Mathematische Annalen, 236(1), 85-93.

[5] Murid A. H. M. and Razali M. R. M., 1999, An integral equation method for conformal mapping of doubly connected regions, Matematika, 15(2), 79-93.

[6] Nasser M. M. S. and Murid A. H. M., 2013, A boundary integral equation with the generalized Neumann kernel for the Ahlfors map, Clifford Analysis, Clifford Algebras and their Applications, 2(4), 307-312.

[7] Nazar K., Murid A. H. M. and Sangawi A. W. K., 2015, Integral equation for the Ahlfors map on multiply connected regions, Jurnal Teknologi, 73(1), 1-9.

[8] Nazar K., Murid A. H. M and Sangawi A. W. K., 2015, Some integral equations related to the Ahlfors map for multiply connected regions, AIP Conference Proceedings, 1691, 1-8.

[9] Bell S. R., 1992, The Cauchy Transform, Potential Theory, and Conformal Mapping, CRC Press, Boca Raton.

[10] Kerzman N. and Trummer M. R., 1986, Numerical conformal mapping via the Szegö kernel, Journal of Computational and Applied Mathematics, 14(1-2), 111-123.

[11] Helsing J. and Ojala R., 2008, On the evaluation of layer potentials close to their sources, Journal of Computational Physics, 227(5), 2899-2921.

[12] Nazar K., Sangawi A. W. K., Murid A. H. M. and Hoe Y. S., 2011, The computation of zeros of Ahlfors map for doubly connected regions, AIP Conference Proceedings, 1750, 1-9.

[13] Nazar K., Murid A. H. M. and Sangawi A. W. K., 2017, The computation of zeros of Ahlfors map for multiply connected regions, AIP Conference Proceedings, 1755, 1-8.

[14] Sangawi A. W. K., 2012, Boundary Integral Equations for Conformal Mappings of Bounded Multiply Connected Regions, [Ph.D. thesis], Universiti Teknologi Malaysia.

[15] Henrici P., 1986, Applied and Computational Complex Analysis Vol. 3, New York: John Wiley and Sons.

[16] Wegmann R., Murid A. H. M. and Nasser M. M. S., 2005, The Riemann-Hilbert problem and the generalized Neumann kernel, Journal of Computational and Applied Mathematics, 182(2), 388-415.

[17] Wegmann R. and Nasser M. M. S., 2008, The Riemann-Hilbert problem and the generalized Neumann kernel on multiply connected regions, Journal of Computational and Applied Mathematics, 214, 36-57.

[18] Nasser M. M. S., Murid A. H. M., Ismail M. and Alejaily E. M. A., 2011, Boundary integral equations with the generalized Neumann kernel for Laplace's equation in multiply connected regions, Applied Mathematics and Computation, 217(9), 4710-4727.

[19] Wahid N. H. A. A., Murid A. H. M. and Muminov, M. I., 2019, Analytical solution for finding the second zero of the Ahlfors map for an annulus region, Journal of Mathematics, 2019, 1-11. 